\title{
Monitoreo forestal con sensoramiento remoto en el marco del cambio climático
}

Forest Monitoring with Remote Sensing Within the Framework of Climate Change

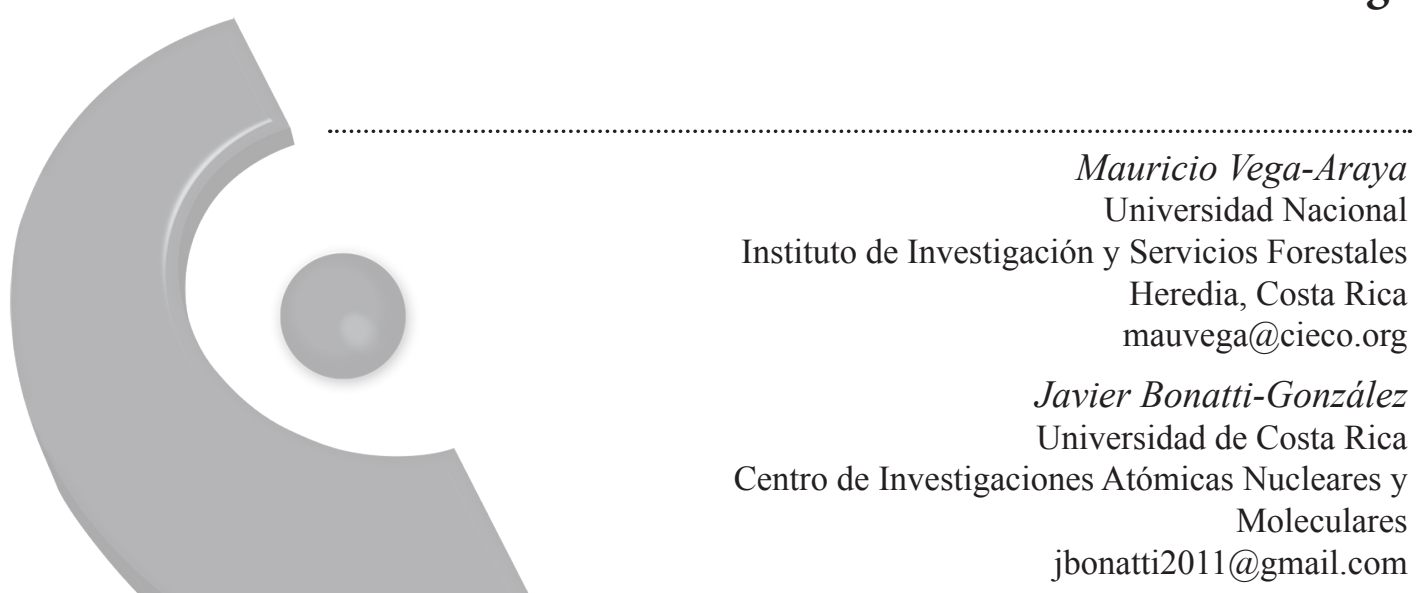

Recibido: 11/09/2014 Aceptado: 30/06/2016

Resumen: La investigación de los efectos del cambio climático es uno de los mayores desafíos para la humanidad. En la actualidad, se debate la cantidad y los flujos de las reservas de carbono en los bosques. En cuanto a estos flujos reducen las concentraciones de $\mathrm{CO}_{2}$ en la atmósfera. En este contexto, la Convención Marco de las Naciones Unidas sobre el Cambio Climático (CMNUCC) se ocupa de las medidas concertadas a nivel internacional para reducir las emisiones de $\mathrm{CO}_{2} \mathrm{y}$ aumentar el secuestro de carbono $(\mathrm{C})$ a través de la intervención humana, incluyendo todas aquellas actividades relacionadas con el manejo y gestión forestal. Sin embargo, la silvicultura y el manejo de bosques han sido reconocidas como actividades donde es extremadamente difícil establecer un control cuantitativo de los aportes inducidos por las actividades del ser humano relativas a la emisión o secuestro de $\mathrm{C}$ a la atmósfera. En las diferentes convenciones y acuerdos, por ejemplo, los acuerdos de Marrakech (2001), solo la re-forestación se incluyó como acciones subvencionables en el contexto del mecanismo de desarrollo limpio (MDL). Luego de arduas negociaciones y con el transcurrir del tiempo, se fueron incorporando una amplia gama de actividades forestales, que 
Revista Universidad en Diálogo • Vol. 6, N. ํㄹ 2, Julio-diciembre 2016, pp. 59-73

ISSN 2215-2849 • EISSN: 2215-4752

DOI: http://dx.doi.org/10.15359/udre.6-2.4

culminaron con los procesos de REDD y REDD+. Dentro de REDD+, por ejemplo, se logró la compensación financiera para las medidas nacionales exitosas que reducen la deforestación y la degradación forestal. Se pensó, entonces, en la necesidad de contar con sistemas eficaces y fiables de seguimiento. Mediante los informes de mediciónreporte y verificación (MRV) se ha establecido y aceptado como un marco estándar para procesos de monitoreo, que consiste en un inventarios de recursos (tradicionalmente inventarios forestales) como un componente clave. En este trabajo se aborda y analizan los retos del sensoramiento remoto en el contexto de los proyectos relacionados con los bosques y el MRV. Se hace, además, un abordaje de la necesidad de estudiar los cambios estacionales de la vegetación en Costa Rica y de cómo estas variaciones pueden afectar los resultados de los proyectos de mapeo de ecosistemas boscosos.

Palabras clave: monitoreo forestal, cambio climático, sensoramiento remoto.

Abstrat: The research of the effects of the climate change is one of the biggest challenges for the humanity. Nowadays, there is a debate about the amount and the flow of the carbon reservation in the forests. As soon as, these flows reduces the concentrations of the $\mathrm{CO} 2$ in the atmosphere. In this context, the UN Framework Convention on Climate Change's deals with concerted actions in an international level to reduce the emissions of $\mathrm{CO} 2$ and increase carbon (C) sequestration through the human intervention, including any activity related with the forest management. However, the forestry and the forest management have been recognized as activities which are extremely difficult to established a quantative control of the contributions induced by activities of the human been relative to the emissions or the carbon sequestration to the atmosphere. In the different conventions and agreements, for example, the agreements Marrakech (2001), only the reforestation was included as an eligible action in the context of the Clean Development Mechanism (CDM). After tough negotiations, and with the passage of time, a range of forest activities were incorporated, culminating with the processes of REDD and REDD+, for example, the financial compensation for the successful national arrangements reducing the deforestation and the forest degradation. It was thought then, about the necessity of counting with effective and reliable systems of tracing. Through the reports of Measuring, Reporting and Verification (MRV) has been established and accepted as an standard framework for monitoiring processes, consisting in a resourse inventory (the tradional forest inventories) as a key component. This work approaches and analyzes the challenges of the remote sensing in the context of the projects related with forest and MRV. In addition, it becomes an approach of the necessity of studying the seasonal changes of the vegetation in Costa Rica and how this variations can affect the results of the projects of mapping the forest ecosystems.

Keywords: climate change, forest monitoring, remote sensing 
Con el comienzo de la industrialización, las actividades humanas han causado el aumento de las concentraciones de $\mathrm{CO}_{2}$ en la atmósfera y han aumentado aproximadamente $280 \mathrm{ppm}$ a más de $390 \mathrm{ppm}$. Esto resulta en un efecto adicional de efecto invernadero que cambia el balance de energía de tierras (Solomon et al., 2007). Si bien es cierto, algunos autores sugieren que se dan cambios en el efecto invernadero por fluctuaciones en la temperatura media de la superficie de la tierra por 2-4 para finales de siglo con efectos regionales en el patrón de las precipitaciones y los fenómenos meteorológicos extremos. Como el $\mathrm{CO}_{2}$ es el principal gas de efecto invernadero antropogénico, es importante tener un conocimiento básico del ciclo global del $\mathrm{CO}_{2}$ cuando se trata de enfoques para la reducción de las concentraciones de $\mathrm{CO}_{2}$ en la atmósfera. La figura 1 muestra la tendencia histórica de los principales grandes almacenes y procesos de carbono, en donde la unidad es el petagramo $(\mathrm{Pg})(1 \mathrm{Pg}=1015 \mathrm{~g}=1 \mathrm{mil}$ millones de toneladas métricas), que es una unidad común para la cuantificación de los depósitos de $\mathrm{C}$ globales. Vemos que, con mucho, la mayor parte de $\mathrm{C}$ se fija en la corteza terrestre, no están disponibles para el intercambio. La porción más grande que es, en principio, disponible para el intercambio, está en los océanos; pero allí, la mayoría del C se encuentra en las sud profundidades, para lo cual, antes de un intercambio con la atmósfera, es necesario un movimiento a las capas superficiales, donde se estima que hay unos $750 \mathrm{Pg}$ de C. Por otro lado, unos $750 \mathrm{Pg}$ se encuentran en la atmósfera y un poco menos en las plantas, principalmente en los árboles. La reserva de carbono en los suelos es de casi tres veces el depósito C. Otra parte importante, en particular en el contexto de los cambios inducidos por el ser humano en la atmósfera, es la liberación de $\mathrm{CO}_{2}$ producto de la utilización de los combustibles fósiles, que incluyen el carbón, el petróleo y el gas. En la figura 1 se ilustra el proceso del balance de carbono. Resaltan dos circunstancias: la primera, el cómo los combustibles fósiles y el cemento tienden siempre a aportar en $\mathrm{CO}_{2}$ y segundo, el hecho de las fluctuaciones entre la superficie terrestre y la atmósfera como sumideros a partir de los años 1950. Esto muestra, a su vez, que los numerosos procesos de intercambio entre los cuatro grandes reservorios de C (la atmósfera, los océanos, la tierra) son un sistema complejo que puede ser bien amortiguado contra intervenciones pequeñas; pero donde es difícil predecir el efecto en intervenciones más grandes. Al parecer, las intervenciones humanas en los últimos 150 años contribuyen a incrementar las fluctuaciones y acentúan la complejidad del sistema. 
Revista Universidad en Diálogo • Vol. 6, N. ํㄹ 2, Julio-diciembre 2016, pp. 59-73

ISSN 2215-2849 • EISSN: 2215-4752

DOI: http://dx.doi.org/10.15359/udre.6-2.4

\section{El papel de los bosques en la dinámica global de carbono}

Los bosques desempeñan un papel vital en el ciclo global del carbono, ya que lo absorben a través de la fotosíntesis y lo secuestran como biomasa, creando así un almacenamiento natural de este: ocupa $1 \mathrm{Gt}$ de carbono cada año, o alrededor del 40 por ciento del total de la absorción terrestre (Britton et al., 2007).

En la figura 1 se ilustran los numerosos procesos de intercambio entre los cuatro grandes reservorios de carbono (la atmósfera, los océanos, la tierra y los combustibles fósiles) y dejan en claro que el ciclo de carbono global es un sistema complejo, que puede amortiguar con intervenciones pequeñas; pero donde es difícil predecir el efecto de las más grandes. Al parecer, las intervenciones humanas, en los últimos 150, pueden considerarse como grandes.

\section{The Global Carbon Budget 1870-2012}

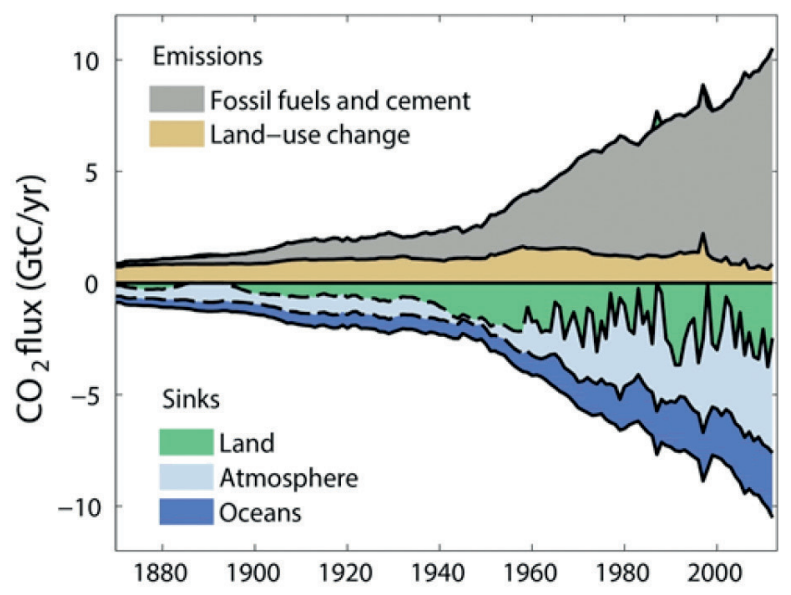

Figura 1. Balance global de carbono. Tomado de: http://shrinkthatfootprint.com/explain-carbon-budget

Los procesos de intercambio de carbono entre el bosque y la atmósfera se dan producto del almacenamiento de carbono durante su crecimiento; sin embargo, existe la liberación de este, debido a los procesos naturales de descomposición. Por eso, en los procesos del bosque, en el largo plazo y sin la intervención humana, este puede ser considerado cercano a un sistema C-equilibrado. Este concepto supone que los árboles, al envejecer, 
pierden gradualmente su vigor y su capacidad de seguir creciendo. Sin embargo, nuevos análisis suponen que los árboles grandes y viejos almacenan siempre carbono (Stephenson et al., 2014).

Cuando el equilibrio se rompe, por ejemplo, con la conversión del bosque a otros usos, esto produce la liberación de $\mathrm{C}$ considerablemente. Lo anterior se da directamente por la liberación de $\mathrm{C}$ por la descomposición de la biomasa, también por el carbono almacenado en los suelos. Sin embargo, cuando la biomasa no se descompone y se utiliza para producir productos a largo plazo, el periodo de liberación se extiende. La biomasa de los bosques en forma de madera también puede sustituir a los combustibles fósiles para la energía, lo que reduce la emisión de $\mathrm{C}$ de los combustibles fósiles.

\section{Sobre el monitoreo de los bosques}

En los Acuerdos de Marakesh (COP7, 2001), los proyectos de carbono forestales solo fueron admitidos para la reforestación y forestación; en ese momento, un acuerdo general sobre el aumento de la captura de carbono por los bosques no se consideró factible ni un control fiable y verificable, por tanto se estimó como imposible. También, se excluyeron los proyectos de "deforestación evitada" a partir del período 2008-2012, por el compromiso con el Protocolo de Kyoto, debido a las preocupaciones de la dilución de las reducciones de los combustibles fósiles, la soberanía y los métodos para medir la reducción de emisiones (Gibbs, Brown, Niles y Foley 2007). Luego, en la COP13 en Bali, el debate sobre el fomento de la deforestación evitada fue recién ascendido y, finalmente, el proceso REDD instalado.

Los procesos políticos son largos y difíciles, y muchas veces parcialmente contradictorios, pues sobreponen los intereses nacionales, que deben ser tomados en cuenta. Por ejemplo, no fue sino hasta los Acuerdos de Cancún en 2010, ${ }^{1}$ cuando los primeros pasos concretos fueron acordados hacia una aplicación amplia de REDD +, que incluye también las declaraciones acerca de la provisión de información y seguimiento. Aunque formulado de una manera aparentemente débil y dejando varias "formas de salir", este es un mensaje claro a los países que deseen participar y, posiblemente, beneficiarse del proceso de REDD +, el cual consiste en crear un sistema nacional de vigilancia forestal robusto y transparente para el seguimiento y la rendición de cuentas. Esto es, en gran parte, una tarea de inventario forestal.

Decisión 1/CP.16: Resultados de la labor del Grupo de Trabajo Especial sobre la Cooperación a Largo Plazo de la Convención FCCC/CP/2010/7/Add.1 
Revista Universidad en Diálogo • Vol. 6, N. ํㄹ 2, Julio-diciembre 2016, pp. 59-73

ISSN 2215-2849 • EISSN: 2215-4752

DOI: http://dx.doi.org/10.15359/udre.6-2.4

En Costa Rica, la fase de diseño del proceso REED se está desarrollando a través del Fondo Nacional de Financiamiento Forestal (FONAFIFO). La estrategia ha instituido un Sistema Nacional de Monitoreo de Bosques (SNMB) que, entre otras tareas, basa su análisis de mediciones -solamenteen los datos que se están obteniendo del Inventario Forestal Nacional (IFN). En la actualidad se está implementando una primera campaña de campo liderada por el Sistema Nacional de Áreas de Conservación (SINAC), con el apoyo económico de GIZ (Deutsche Gesellschaft für Internationale Zusammenarbeit, por sus siglas en alemán). Por lo tanto, el SINAC tiene el compromiso de estimar el carbono por tipo forestal a partir del IFN, y reportarlo, bianualmente, al Instituto Meteorológico Nacional (IMN) y a la Secretaría REDD+. Dentro de estos procesos, sin embargo, debe reconocerse el valor que tiene la investigación a largo plazo a través del monitoreo de los cambios en vegetación en procesos que integren la información de campo como, por ejemplo, redes de Parcelas Permanentes de Muestreo (PPM).

\section{Monitoreo}

Confusiones de clase son el problema más frecuente en la clasificación de ecosistemas usando el sensoramiento remoto. En las zonas tropicales, la vegetación verde es un espectro muy complejo de composiciones diferentes de plantas y de variación estacional. La Reducción de Emisiones por Deforestación y Degradación de los Bosques (REDD y REDD +) es el mercado/iniciativa financiera cuya idea fundamental es reducir las emisiones de gases de efecto invernadero provenientes de la deforestación y la degradación de los bosques. El éxito de toda iniciativa REDD+ depende de que demuestren que poseen un sistema de monitoreo (que incluya medición), reporte y verificación. Parte del desafío en proyectos REDD+ consiste en cómo diferenciar la deforestación y la degradación, la cual está profundamente relacionada con la cuestión de cómo medir cada una. Sin embargo, ambos conceptos están conectados en la dimensión político-económica y científica. Además de esto, todos los actores necesitan una base para negociar o aplicar a una iniciativa específica. Normalmente, esta base es un mapa a escala nacional del bosque.

También, en el marco de los procesos políticos internacionales, como el Convenio sobre la Diversidad Biológica (CDB) y la Convención sobre el Cambio Climático, el monitoreo estacional y la fragmentación del bosque llaman la atención de los tomadores de decisión. Ha sido objeto de una intensa investigación durante las últimas décadas (por ejemplo, Lindenmayer, Cunningham, Donnelly y Lesslie, 2002; Millington, Velez-Liendo y Bradley, 2002; Riitters et al., 2002). 
Para producir un mapa del bosque en el tiempo de forma regular y a nivel o escala nacional, solamente el sensoramiento remoto puede dar, a un costo razonable, la información necesaria. Además de lo anterior, el sensoramiento remoto juega un papel importante en aquellos casos donde no es posible llevar a cabo inventarios terrestres, por ejemplo, en áreas de difícil o imposible acceso, ya sea por su ubicación remota o por cuestiones de seguridad.

Las plataformas remotas proporcionan el único medio de ver una gran parte de la superficie de la tierra a intervalos regulares. Por medio de la absorción selectiva y la reflectancia de la luz por las plantas, los sensores ópticos permiten recoger grandes cantidades de información (Ollinger, 2011) así como proporcionar información esencial para el clima y los modelos de ecosistemas (Ustin, Roberts, Gamon, Asner y Green, 2004).

La fragmentación de los bosques ha dado lugar a la interrupción de los procesos ecológicos y, en muchos casos, a la degradación de los recursos naturales. Esto hace que el recurso del sensoramiento sea relevante para la política forestal y de conservación, ya que es uno de los principales indicadores para el manejo forestal sostenible en la escala de paisaje, donde se asume una relación entre la fragmentación y la conservación ecológica, y los problemas económicos.

Para evaluar la fragmentación de los bosques y su relación con los procesos ecológicos, la cuantificación es la demanda. Si bien no existe un enfoque intuitivo o inmediato, varias métricas de fragmentación han propuesto que se calculan a partir de una combinación de variables directamente mensurables, tales como el área de parche, longitud de borde, número de parches, dominancia, diversidad, dimensión fractal y otros. Para estos efectos, se han desarrollado software especializados, por ejemplo, Fragstats (McGarigal, Cushman, Neel y Ene, 2002), APACK (Mladenoff y DeZonia, 2004), Patch Analyst (Elkie, Rempel y Carr, 1999) y r.le en el entorno HIERBA (Baker y Cai, 1992) para calcular una variedad de índices o indicadores. Esto ha llevado a un aumento en el análisis cuantitativo del paisaje en los últimos años. Cabe anotar que muchas de estas herramientas informáticas hoy son de acceso libre.

Sin embargo, estudios de métricas de fragmentación han atraído menos atención en la bibliografía científica que el desarrollo de nuevas métricas (Millington et al., 2002), aunque, como con cualquier otro indicador, esta sensibilidad es una pieza importante de información para el monitoreo del bosque. En general, los factores de relevancia para el cálculo e interpretación de indicadores de monitoreo del bosque son: 
Revista Universidad en Diálogo • Vol. 6, N. ํㄹ 2, Julio-diciembre 2016, pp. 59-73

ISSN 2215-2849 • EISSN: 2215-4752

DOI: http://dx.doi.org/10.15359/udre.6-2.4

1. Resolución espacial

2. Resolución temporal (estacionalidad)

3. Definición de clase

4. Delimitación

5. Topografía

Bosques tropicales y subtropicales están bajo interés especial, por motivos de conservación y su relación con el cambio climático, ya que son aristas para la biodiversidad y captura de carbono. Sin embargo, al mismo tiempo, se enfrentan a grandes amenazas de ser convertidos en otros sistemas de uso de la tierra. El sensoramiento remoto es una de las tecnologías clave para el monitoreo de la cubierta forestal y las evaluaciones de la fragmentación del bosque, ya que ofrece la posibilidad de monitorear grandes áreas con un esfuerzo razonable. Actualmente, una cantidad de sistemas de clasificación de la cubierta terrestre no toman en cuenta la estacionalidad, es decir, los cambios fisionómicos debidos a la disponibilidad de agua o a la actividad fotosintética de las hojas de los árboles. Además, la mayoría de ellos no son aplicables a una clase específica o la definición de límites no está basada en criterios cuantitativos. Por lo tanto, la comparación de los mapas de cobertura del suelo y las métricas derivadas de la fragmentación en el espacio y el tiempo de diferentes iniciativas o proyectos específicos, a menudo, no es posible.

En los países en desarrollo como Costa Rica, los recursos naturales son limitados y se agotan cuando se utilizan para el desarrollo de actividades productivas. Con la premisa de optimización de recursos escasos, es indudable que la adquisición de información de forma ágil y oportuna sea una herramienta para la planificación en todo lo relacionado al ambiente.

El sensoramiento remoto puede jugar un papel importante en el seguimiento y cuantificación de las reservas de carbono en biomasa (Fagan y DeFries, 2009) y actividades de secuestro; esta tecnología podría tener muchas ventajas como la observación global, cobertura repetitiva, varios tipos de sensores y, en ocasiones, implementación de bajo costo. La teleobservación se ha demostrado que es una herramienta para la vigilancia del medio ambiente, especialmente en los países menos desarrollados.

En ecosistemas tropicales, la identificación de especies o grupos de especies es importante para entender y ayudar en las clasificaciones de manera más detallada $\mathrm{y}$, además, para evitar confusiones de clase cuando se utiliza el sensoramiento remoto. Métodos fiables para el 
sensoramiento remoto de la composición del bosque a nivel de especie serían un avance importante hacia la comprensión de la dinámica y procesos fisiológicos. Aunque varios estudios han logrado importantes avances, los desafíos siguen siendo considerables en cuanto a los métodos estandarizados para la generación de la información a nivel de especie de paisaje. Estudios que incluyan la dinámica de los cambios en el tiempo no están bien desarrollados en la actualidad.

Además, los datos obtenidos por sensoramiento remoto se han convertido en la principal fuente para la estimación de la biomasa a nivel de paisaje (por ejemplo, Asner, 2010; González et al., 2010). Sin embargo, se ha demostrado que la estimación de la biomasa sigue siendo una tarea difícil (Andersson and K., 2009), sobre todo en aquellas áreas de estudio con complejas estructuras como los bosques tropicales.

El uso de datos de los sensores ópticos multiespectrales (5 a 15 bandas) resultan más adecuados para los sitios de bosques con estructuras de bosque relativamente simples, como es el caso de las plantaciones forestales. Sin embargo, para estudiar la combinación de respuestas espectrales y texturas de imagen se necesitan los datos hiperespectrales (más de 200 bandas) que permitan una adecuada selección de las respuestas espectrales y el desarrollo de algoritmos (índices) para la estimación de las variables de interés a diferentes escalas.

Un ejemplo de un sensor multiespectral es el ampliamente utilizado Landsat Thematic Mapper (TM), del cual se han elaborado numerosas aplicaciones de observación de la tierra. En general, el número relativamente pequeño de canales de adquisición que caracteriza sensores multiespectrales puede ser suficiente para discriminar entre diferentes clases de cubierta terrestre (por ejemplo, la silvicultura, el agua, los cultivos, las zonas urbanas, etc.).No obstante, su capacidad de discriminación es muy limitada cuando diferentes tipos (o condiciones) de la misma especie (por ejemplo, diferentes tipos de bosque) han de ser reconocidas. Los sensores hiperespectrales se pueden utilizar para tratar este problema. Estos sensores se han caracterizado tradicionalmente por tener una resolución espectral muy alta, empero, en la actualidad, con el uso de misiones aerotransportadas, se puede obtener también una alta resolución espacial.

Otra forma de estudiar los patrones de reflectividad es mediante la colección de firmas espectrales. Las firmas espectrales representan 
Revista Universidad en Diálogo • Vol. 6, N. ํㄹ 2, Julio-diciembre 2016, pp. 59-73

ISSN 2215-2849 • EISSN: 2215-4752

DOI: http://dx.doi.org/10.15359/udre.6-2.4

reflectancia típica de la formación (formaciones de árboles, por ejemplo o de hojas individuales), en una variedad de longitudes de onda del espectro electromagnético. Las firmas espectrales ayudan a validar los datos obtenidos de los sensores instalados en satélites como en aviones. Con esto se localizan los objetos y se obtienen mejores resultados de las mediciones. Algoritmos desarrollados a través de espectroscopia deben ser más fáciles para controlar los cambios y obtener cálculos precisos.

La cubierta vegetal, como otros tipos de cobertura, muestra comportamientos espectrales específicos, por lo tanto, la espectroscopia aplicada contribuye a la generación de índices específicos de vegetación. Aunque hay una serie de índices disponibles, su funcionalidad depende de las condiciones del sensor y la influencia atmosférica. Al hacer ajustes locales con espectrómetros de campo, es posible generar algoritmos particulares para estudios específicos.

La espectroscopia de campo puede utilizarse junto con los sensores remotos en, al menos, tres componentes: 1) Desarrollo y prueba de modelos de reflectancia espectral (mediciones espectrométricas se pueden utilizar para la selección de las bandas más adecuadas para ser utilizadas por un sensor específico, por ejemplo). 2) Recoger y calibrar datos obtenidos por satélite y la información de las misiones aerotransportadas. 3) En una variedad de aplicaciones (por ejemplo, se utiliza comúnmente en la agricultura, para los cultivos, y en estudios de gases de efecto invernadero relacionados con la pigmentación o la nutrición, sobre la base de las características espectrales de las hojas).

La cuantificación significativa del bosque en términos de seguimiento es relevante, entre otros, para la investigación de sus valores ecológicos (biodiversidad) y las consecuencias de su sostenibilidad.

Costa Rica ha impulsado un Programa de Monitoreo Ecológico Terrestre de las Áreas Protegidas y Corredores Biológicos de Costa Rica (PROMEC-CR). Relacionado con los bosques, define que la evaluación de la estructura y composición del hábitat sobre la base de datos de sensores remotos debe ser la columna vertebral del programa de monitoreo a escala nacional.

Sin embargo, la cuantificación de los bosques y otros ecosistemas a través del tiempo con sensoramiento remoto es sensible a factores como: 1) La información disponible en cuanto a su resolución: espacial, la resolución radiométrica, espectral y temporal. 2) Costo. 3) Tiempo de transformación. 
4) Sistema de clasificación y definición de clase. 5) Definición de límite. Por otro lado, excelentes sensores pasivos (en términos de estos aspectos, incluyendo correcciones topográficas) podrían ser deficientes cuando, por lo menos, hay 10 o 20 por ciento de cobertura de nubes, en el caso de algunas regiones de Costa Rica.

\section{En términos más concretos: ¿qué se necesita?}

Con el fin de entender la dinámica del carbono en los bosques y el papel de los bosques en el ciclo global del carbono, y generar una base de información que se pueda utilizar para los esquemas de financiamiento de carbono existentes y futuros, se requieren datos fiables para describir las reservas de carbono y su dinámica con un cierto grado de precisión y claridad espacial. En términos más concretos, para todas las categorías de uso de la tierra vistos como depósitos de carbono o bien emisores de este, necesitan datos que deben elaborarse para los informes nacionales a los procesos respectivos. Los datos de actividad y factores de emisión deben ser determinados y reportados. "Los datos de actividad" se refieren a la zona y al área cambiante de la tierra o a los tipos de terrenos forestales de bosque. Los "factores de emisión" se refieren a la densidad de carbono por unidad de superficie de bosque. Ambas variables son variables típicas del inventario forestal, aunque la terminología es ligeramente diferente. Los cambios en la superficie forestal son relevantes para informar UNFCCC (The United Nations Framework Convention on Climate Change, por sus siglas en inglés) cuando son consecuencia de las actividades humanas; lo mismo vale para los factores de emisión. Es decir, para las dos variables, lo cual no solo es importante para el estado de grabación y cambios, sino también para establecer pruebas de la intervención humana.

Los inventarios forestales nacionales, ya que se establecieron en muchos países, pueden proporcionar una gran parte de la información requerida; aún más, están diseñados de una manera adecuada. Estos se constituyen en las mismas fuentes de datos valiosos de tiempo, pues aportan elementos de la biodiversidad, de la utilización de los recursos forestales, del recurso de árboles fuera del bosque y de varios otros dominios.

Las partes que deseen participar en el proceso de REDD + deben adherirse a los estándares definidos: dar pruebas de que, de hecho, han logrado los objetivos de gestión de carbono que ellos desean "vender". Este "prestar declaración" requiere transparencia de los métodos y aplicación. El estándar es 
Revista Universidad en Diálogo • Vol. 6, N. ํㄹ 2, Julio-diciembre 2016, pp. 59-73

ISSN 2215-2849 • EISSN: 2215-4752

DOI: http://dx.doi.org/10.15359/udre.6-2.4

ahora MRV (Medición - Informes - Verificación), que es un instrumento que se está aplicando en numerosos procesos políticos. La adhesión a los principios de MRV es esencial para todos los países que participan en REDD +.

Medición, reporte y verificación (MRV) está incluido en los Acuerdos de Cancún como uno de los elementos más críticos necesarios para la implementación exitosa de cualquier mecanismo REDD + (IISD, 2011). El Programa ONU-REDD ofrece una definición concisa de MRV (GOFC-GOLD, 2009), donde se recalcan, además, los objetivos globales de reducción de carbono y los elementos pertinentes de MRV (esencialmente $\mathrm{M}=$ medición y seguimiento) para apoyar los procesos correspondientes. En este queda claramente establecido que los inventarios forestales nacionales y sensoramiento remoto desempeñan un papel crucial.

Es preciso, entonces, el establecer procesos de recolección de datos (medición) a través del tiempo, proporcionando información básica, incluyendo exactitud y la precisión asociada para el rango de las variables relevantes. Posibles fuentes de datos son las mediciones de campo, observaciones de campo y puntos de control, la interpretación sistemática de sensores remotos. La interpretación sistemática en el caso del uso del sensoramiento remoto se refiere a tomar en cuenta las variaciones estacionales y las diferencias por el uso de sensores diferentes.

El proceso de presentación de informes (reporte) debe corresponder a los formatos predeterminados y según las normas establecidas, en especial el Grupo Intergubernamental de Expertos sobre el Cambio Climático (IPCC) y Guía de Buenas Prácticas. Por último la verificación como rutina formal de los que permita mejorar las comunicaciones nacionales y los informes de los inventarios nacionales.

Es importante, para los actores en el monitoreo forestal, comprender los principios y el papel de la MRV donde se encuentra la base para los mecanismos y la puesta en funcionamiento de MRV para REDD. Una serie de cuestiones técnicas quedan, sin embargo, por tratar, aunque han sido discutidas desde hace décadas. Por ejemplo, en el marco de los inventarios forestales tradicionales, la cuestión de la definición de los bosques y el equilibrio entre precisión y costo cuando se mira en los objetivos generales. La incertidumbre y su cuantificación son elementos relevantes y cruciales en todas las actividades de inventario y monitoreo. El costo del MRV es, por supuesto, la cuestión principal: si un sistema de MRV para vigilar el cumplimiento es prohibitivamente caro, todo el proceso REDD+ podría ser una crónica de una muerte anunciada. 


\section{Conclusiones}

Hay una larga tradición en la toma de datos sobre los bosques y, en general, sobre inventarios forestales que se remontan varios siglos. Sin embargo, cuando se trata del proceso de REDD+, se vuelve muy claro que el monitoreo forestal tiene algo más que una dimensión científico-técnica, la cual es la dimensión política. En el contexto de REDD+ de la información proporcionada por los sistemas de monitoreo, tendrá una implicación en lo económico y en la consolidación de ecosistemas boscosos. Por ello, la optimización técnico-científica de los procesos de monitoreo debe ir de la mano con el seguimiento de los procesos políticos. Lo anterior es todo un reto para el monitoreo forestal que debe cerrar la brecha entre ciencia y política. Significa, además, el involucramiento serio y sostenido de la academia desde el proceso de enseñanza aprendizaje y la formación de nuevas generaciones de profesionales que integren mejor la dimensión técnico-científica con la política.

\section{Referencias}

Andersson, K. T. E., \& K., R. (2009). National forest carbon inventories: Policy needs and assessment capacity. Climate Change, 93(1), 69-101.

Asner, G. (2010). Forest carbon, biodiversity, and redd+: Opportunity and challenge .

Baker, W. and Cai, Y. (1992). The r.le programs for multiscale analysis of landscape structure using the grass geographic information system. Landscape Ecology, 7, 291-302.

Britton, S.B., Gurney, K. R., Tans, P. P., Sweeney, C., Peters, W., Bruhwiler, L., Ciais, P., Ramonet, M., Bousquet, P., Nakazawa, T., Aoki, S., Machida, T., Inoue, G., innichenko, N., Armin, J.L., Jordan, Heimann, M., Shibistova, O., Langenfelds, R. L., Steele, L. P., and Denning, S.A. (2007). Weak Northern and Strong Tropical Land Carbon Uptake from Vertical Profiles of Atmospheric CO2. Science, 316, 1732-1734.

Elkie, P., Rempel, R., and Carr, A. (1999). Patch Analyst User's Manual. Ont. Min. Natur. Resour. Northwest Sci. and Technol. Thunder Bay, Ont. TM-002.

Fagan, M. and DeFries, R. (2009). Measurement and monitoring of the worlds forests: A review and summary of remote sensing technical capability, 2009-2015. Rff report, Columbia University. 
Revista Universidad en Diálogo • Vol. 6, N. ํㄹ 2, Julio-diciembre 2016, pp. 59-73

ISSN 2215-2849 • EISSN: 2215-4752

DOI: http://dx.doi.org/10.15359/udre.6-2.4

Gibbs, H. K., Brown, S., Niles, J., and Foley, J. A. (2007). Monitoring and estimating tropical forest carbon stocks: making redd a reality. Environmental Research Letters, 2, 1-13.

GOFC-GOLD. (2009). Reducing greenhouse gas emissions from deforestation and 46 degradation in developing countries: A sourcebook of methods and procedures 47 for monitoring, measuring and reporting, gofc-gold report version cop14-2, 48. Technical report, GOFC-GOLD Project Office, Natural Resources Canada, Alberta, Canada.

González, P., Asner, Gregory P., Battles, J. J., Lefsky, M. A., Waring, K. M., and Palace, M. (2010). Forest carbon densities and uncertainties from lidar, quickbird, and field measurements in california. Remote Sensing of Environment, 114, 1561-1575.

Gullison, R. E., Frumhoff, P., Canadell, J., Fiel, C., Nepstad, D., Hayhoe, K., Avissar, R., Curran, L., Friedlingtein, P., Jones, C., and Nobre, C. (2007). Tropical forests and climate policy. Science, 316, 885-986.

IISD (2011). Measurement, reporting and verification (MRV). On line.

Lindenmayer, D., Cunningham, R., Donnelly, C., and Lesslie, R. (2002). On the use of landscape surrogates as ecological indicators in fragmented forests. Forest Ecology and Management, 159, 203-216.

McGarigal, K., Cushman, S., Neel, M., and Ene, E. (2002). Fragstats v3: Spatial pattern analysis program for categorical maps. .Recuperado de: www.umass.edu/landeco/research/fragstats/fragstats.html.

Millington, A., Velez-Liendo, X., and Bradley, A. (2002). Scale dependence in multitemporal mapping of forest fragmentation in bolivia: implications for explaining temporal trends in landscape ecology and applications to biodiversity conservation. ISPRS Journal of Photogrammetry and Remote Sensing, 57, 289-299.

Mladenoff, D. and DeZonia, B. (2004). Apack 2.23 analysis software.Recuperado de: http://flel.forest.wisc.edu.

Ollinger, S. V. (2011). Sources of variability in canopy reflectance and the convergent properties of plants. New Phytologist, 189(2), 375-394.

Riitters, K., Wickham, J., O’Neill, R., Jones, K., Smith, E., Coulston, J., Wade, T., and Smith, J. (2002). Fragmentation of continental united states forests. Ecosystems, 5, 815-822.

Solomon, S., Qin, D., Manning, M.., Chen, Z., Marquis, M., Averyt, K., Tignor, M., and M. H. (edits.) (2007). Contribution of Working Group I to the Fourth Assessment Report of the Intergovernmental Panel on Climate Change. Cambridge Univ. Press. 
Stephenson, N. L., Das, A. J., Condit, R., Russo, S. E., Baker, P. J., Beckman, N. G., Coomes, D. A., Lines, E. R., Morris, W. K., Rueger, N., Alvarez, E., Blundo, C., Bunyavejchewin, S., Chuyong, G., Davies, S. J., Duque, A., Ewango, C. N., Flores, O., Franklin, J. F., Grau, H. R., Hao, Z., Harmon, M. E., Hubbell, S. P., Kenfack, D., Lin, Y., Makana, J.-R., Malizia, A., Malizia, L. R., Pabst, R. J., Pongpattananurak, N., Su, S.-H., Sun, I.-F., Tan, S., Thomas, D., van Mantgem, P. J., Wang, X., K., W. S., and Zavala, M. A. (2014). Rate of tree carbon accumulation increases continuously with tree size. Science, 507, 90-93.

Ustin, S. L., Roberts, D. A., Gamon, J.A., Asner, G. P., and Green, R. O. (2004). Using Imaging Spectroscopy to Study Ecosystem Processes and Properties. BioScience, 54(6), 523-534. 